\title{
Correspondence
}

\section{Nebulizer, Inhaled Remdesivir Nanoparticle Co-administered with Withania Somnifera may Minimize the Hepatotoxicity in COVID-19}

\author{
R. Velmurugan', P. Manoj Kumar², Keerthi G.S. Nair ${ }^{2 *}$ \\ ${ }^{1}$ Department of Pharmacology, School of Pharmaceutical Sciences, Vels Institute of Science Technology and Advanced Studies, Chennai 600117, India \\ ${ }^{2}$ Department of Pharmaceutics, The Dale View College of Pharmacy and Research Centre, Punalal, Poovachal, Trivandrum, Kerala 695575, India
}

A RTI CLE INF O
Article History
Received 17 March 2021
Accepted 05 July 2021
Keywords
COVID-19
remdesivir
hepatotoxicity
nanoparticle
target delivery
ashwagandha
improved clinical outcome

\begin{abstract}
Remdesivir is a broad spectrum antiviral agent meant to inhibit viral RNA polymerases against members of several virus families, including filoviruses. Although reported for significant clinical improvements in COVID-19, a detailed observation of the connection with the drawbacks of remdesivir therapy was poor pulmonary distribution and hepatotoxicity (transaminase elevation) was recorded that resulted in discontinuation of remdesivir therapy. Nanodrug delivery facilitates a large drug payload to the targeted site improving the efficacy. With the lesser dose administered, could be expected for minimized adverse events. Additional payload of ashwagandha will protect the liver from the damages caused if any. Remdesivir in nanoformulation may be a safe and an effective therapeutic agent for COVID 19 when co-adminstered with ashwagandha.
\end{abstract}

(C) 2021 The Authors. Published by Atlantis Press B.V. This is an open access article distributed under the CC BY-NC 4.0 license (http://creativecommons.org/licenses/by-nc/4.0/).

\section{INTRODUCTION}

The world is gearing up to battle against the Coronavirus Disease 19 (COVID-19) outbreak which has already led to more than 2,424,060 deaths and 109,594,835 confirmed cases globally as on February 18, 2021 [1]. There are currently no approved effective therapeutic agents available for the treatment of COVID-19. National Institutes of Health, United States says it has begun a clinical trial testing the ability of Gilead's experimental drug remdesivir to treat people with COVID-19. The news comes at a crucial moment. Remdesivir is a prodrug of a nucleotide analogue that is intracellularly metabolized to an analogue of adenosine triphosphate that inhibits viral RNA polymerases. Remdesivir has broadspectrum activity against members of several virus families, including filoviruses (e.g., Ebola) and coronaviruses [2].

Remdesivir is a broad spectrum antiviral agent meant to inhibit viral RNA polymerases against members of several virus families. It is given as an infusion into a vein. It must be given slowly, and the infusion can take 30-120 min to complete. It is usually given once per day for 5-10 days. Remdesivir exhibited cell culture antiviral activity against a clinical isolate of SARS-CoV-2 in primary human airway epithelial cells with a 50\% effective concentration $\left(\mathrm{EC}_{50}\right)$ of $9.9 \mathrm{nM}$ after $48 \mathrm{~h}$ of treatment. The $\mathrm{EC}_{50}$ values of remdesivir against Severe Acute Respiratory Syndrome-related CoronaVirus (SARS-CoV-2) in Vero cells was $137 \mathrm{nM}$ at $24 \mathrm{~h}$ and $750 \mathrm{nM}$ at $48 \mathrm{~h}$

"Corresponding author. Email: keerthinair.dr@gmail.com post-treatment [3,4]. Many clinical studies have reported a significant clinical improvement with the transaminase elevations in the remdesivir clinical development program, including in healthy volunteers and patients with COVID-19, without evidence of clinical hepatitis and elevated hepatic enzymes resulted in discontinuation of remdesivir therapy $[5,6]$. As transaminase elevations have been reported as a component of COVID-19 in some patients, discerning the contribution of remdesivir to transaminase elevations in this patient population is challenging.

Following administration, drug is distributed to all the parts and that the total dose, rather than pulmonary distribution, is related to the clinical effect. A $10 \mathrm{mg} / \mathrm{kg}$ intravenous dose given to cynomolgus monkeys distributes to the testes, epididymis, eyes, and brain within $4 \mathrm{~h}$ with a limited pulmonary distribution [7].

Remdesivir for compassionate use is provided in two dosage forms, a solution formulation and a lyophilized formulation. However selecting the optimal formulation for a drug is crucial to ensure its clinical success. Considering the frequency of liver dysfunction in patients with COVID-19, attribution of hepatotoxicity to either remdesivir or the underlying disease is challenging.

Detailed observation of the connection with the drawbacks of remdesivir therapy that poor pulmonary distribution and hepatotoxicity, nanodrug delivery of remdesivir co-administered with ashwagandha, an Indian herb reported for its hepatoprotective potentials, could be an ideal choice to treat COVID-19 proving to be safe and effective. 
Alveolar epithelial cells are an important target for coronavirus infection in the lung. In the alveolar region, the size of the particles appears to dominate the clearance rate. Micron scale particles between 1 and $5 \mu \mathrm{m}$ are efficiently taken up by macrophages and particles $>6 \mu \mathrm{m}$ are generally not phagocytosed but rather exhaled. Particles at the nanoscale ( $\leq 200 \mathrm{~nm}$ ) can cross the cellular barrier independent of energy and are phagocytosed by macrophages [8]. This suggests that particles that are small enough can evade macrophage clearance when deposited in the deep lung. As a result, nanosized particles have ideal deposition characteristics for the delivery of remdesivir to alveolar regions of the lung to inhibit the viral RNA polymerase.

Extravasation of remdesivir given as an infusion into a vein could be bypassed by pulmonary administration, specifically nanoparticle inhalation. This route avoids exposure to the systemic ciruculation prior to the lung delivery, thus avoiding hepatic first pass metabolism and increasing the delivery of nanoparticles to the lung as compared with intravenous delivery.

On the other hand, application of remdesivir through infusion may end up in non-specific distribution and to prevent the same, nanoparticle could be added with targeting moieties to their surface to direct their delivery. The targeting moiety will interact with molecules on the target cell's surface, i.e. alveoli's surface. Thus, targeted remdesivir nanoparaticle must be engineered with a targeting moiety (antibodies, glucose, transferin, folate etc.) allowing the moiety to interact with the alveoli cell surface, making it to overcome the barrier to nanoparticle uptake.

It was found the upper respiratory tract is the most prevalent site of COVID-19 infection early in disease. Delivering drugs directly to the primary site of infection with a nebulizer, inhaled nanoparticle formulation may enable more targeted and accessible administration in patients and potentially lower systemic exposure to the drug.

Drugs largely bypass metabolism when directly delivered into the lung and therefore a lesser dose of remdesivir is sufficiently enough if delivered right in the lungs to inhibit the viral RNA polymerase and this is possible with nebulizer, inhaled nanoparticle formulation. The adverse events (low blood pressure, nausea, vomiting, sweating, increases in levels of liver enzymes) [3] noted with the loading dose of remdesivir $200 \mathrm{mg}$ once daily in patients, followed by a maintenance dose of $100 \mathrm{mg}$ once daily may be expected to minimize upon administration of much lesser dose of remdesivir with a nebulizer, inhaled nanoparticle formulation.

At this progressive state of COVID-19 pandemic, the therapeutic gaps in conventional management have highlighted the need for the integration of traditional knowledge systems with modern medicine. Ayurvedic medicines, especially Ashwagandha (Withania somnifera), may be beneficial in the management of COVID-19. Withania somnifera is a widely prescribed Ayurvedic botanical known as hepatoprotective, immunomodulatory, antiviral, antiinflammatory, and adaptogenic agent. Attribution of hepatotoxicity to either remdesivir or the underlying disease is uncertain. To minimize the toxicity and to enhance the therapeutic efficacy, remdesivir could be administered as nebulizer inhaled nanoparticle once per day for 5-10 days following the oral administration of $W$. somnifera at a dose of $500 \mathrm{mg}$ per day for 6 weeks [9]. Many preclinical and clinical study results clearly demonstrates that
W. somnifera possesses promising hepatoprotective effects through its antioxidant activity and hence suggested its use as a potential therapeutic agent for hepatic disorders [10-15].

\section{CONCLUSION}

To conclude, with the benefits of nano drug delivery that includes, nometric size permits drug delivery through impermeable barriers, large surface area to volume ratios for large drug payloads incorporation and improved efficacy, enhancing stability and bioavailability, increased specificity, improved antiviral delivery and controlled drug release to the target through engineered moieties, decrease the emergence of drug resistance, personalized therapy possibility, protection of the drugs and low adverse drug side effects, remdesivir nanopartilces may be a safe and an effective therapeutic agent for COVID-19 when co-adminstered with $W$. somnifera.

\section{CONFLICTS OF INTEREST}

The authors declare they have no conflicts of interest.

\section{AUTHORS' CONTRIBUTION}

RV and KGSN conceived of the presented idea; PMK prepared the manuscript.

\section{ACKNOWLEDGMENTS}

The authors would like to thank Vels Institute of Science Technology and Advanced Studies, Chennai and the Dale View College of Pharmacy and Research Centre, Trivandram for the motivations given in sharing our contributions toward the treatment options of COVID-19.

\section{REFERENCES}

[1] WHO Coronavirus (COVID-19). Available from: https:// covid19.who.int (accessed February 19, 2021).

[2] de Wit E, Feldmann F, Cronin J, Jordan R, Okumura A, Thomas T, et al. Prophylactic and therapeutic remdesivir (GS-5734) treatment in the rhesus macaque model of MERS-CoV infection. Proc Natl Acad Sci U S A 2020;117:6771-6.

[3] Fact sheet for healthcare providers: emergency use authorization of remdesivir. Available from: https://www.fda.gov/ media/137566/download.

[4] Sheahan TP, Sims AC, Zhou S, Graham RL, Pruijssers AJ, Agostini $\mathrm{ML}$, et al. An orally bioavailable broad-spectrum antiviral inhibits SARS-CoV-2 in human airway epithelial cell cultures and multiple coronaviruses in mice. Sci Transl Med 2020;12:eabb5883.

[5] Lescure FX, Bouadma L, Nguyen D, Parisey M, Wicky PH, Behillil S, et al. Clinical and virological data of the first cases of COVID-19 in Europe: a case series. Lancet Infect Dis 2020;20: 697-706.

[6] Grein J, Ohmagari N, Shin D, Diaz G, Asperges E, Castagna A, et al. Compassionate use of remdesivir for patients with severe Covid-19. N Engl J Med 2020;382:2327-36. 
[7] Warren TK, Jordan R, Lo MK, Ray AS, Mackman RL, Soloveva V, et al. Therapeutic efficacy of the small molecule GS-5734 against Ebola virus in rhesus monkeys. Nature 2016;531:381-5.

[8] Carvalho TC, Peters JI, Williams RO. Influence of particle size on regional lung deposition - what evidence is there?. Int J Pharm 2011;406:1-10.

[9] Mohan A, Menon A, Chacko J, Mohan P, Robin DT. An eye into the allegations about ashwagandha. Liver Int 2020;40:2034-5.

[10] Prince Sabina E, Rasool M, Vedi M, Navaneethan D, Ravichander $\mathrm{M}$, Parthasarthy $\mathrm{P}$, et al. Hepatoprotective and antioxidant potential of Withania somnifera against paracetamol-induced liver damage in rats. Int J Pharm Pharm Sci 2013;5:648-51.

[11] Devkar ST, Kandhare AD, Zanwar AA, Jagtap SD, Katyare SS, Bodhankar SL, et al. Hepatoprotective effect of withanoliderich fraction in acetaminophen-intoxicated rat: decisive role of TNF- $\alpha$, IL- $1 \beta$, COX-II and iNOS. Pharm Biol 2016;54: 2394-403.
[12] Kumar R, Rai J, Kajal NC, Devi P. Comparative study of effect of Withania somnifera as an adjuvant to DOTS in patients of newly diagnosed sputum smear positive pulmonary tuberculosis. Indian J Tuberc 2018;65:246-51.

[13] Harikrishnan B, Subramanian P, Subash S. Effect of Withania somnifera root powder on the levels of circulatory lipid peroxidation and liver marker enzymes in chronic hyperammonemia. J Chem 2008;5:872-7.

[14] Khalil HMA, Eliwa HA, El-Shiekh RA, Al-Mokaddem AK, Hassan M, Tawfek AM, et al. Ashwagandha (Withania somnifera) root extract attenuates hepatic and cognitive deficits in thioacetamide-induced rat model of hepatic encephalopathy via induction of Nrf2/HO-1 and mitigation of NF- $\mathrm{\kappa B} / \mathrm{MAPK}$ signaling pathways. J Ethnopharmacol 2021;277:114141.

[15] Xia Y, Wang P, Yan N, Gonzalez FJ, Yan T. Withaferin A alleviates fulminant hepatitis by targeting macrophage and NLRP3. Cell Death Dis 2021;12:174. 